\title{
Title: Haematological management of major bleeding associated with direct oral anticoagulants - UK experience
}

Laura Green, ${ }^{* 1,2,3}$ Joachim Tan, ${ }^{* 1}$ Sotiris Antoniou, ${ }^{1}$ Raza Alikhan, ${ }^{4}$ Nicola Curry, ${ }^{5,6}$ Tamara Everington, ${ }^{7,8}$ Khalid Saja, ${ }^{9}$ Simon Stanworth, ${ }^{5,6,10}$ Campbell Tait, ${ }^{11}$ Joan K Morris, ${ }^{1}$ Peter MacCallum ${ }^{1,2}$

*Joint first authors

${ }^{I}$ Barts and the London School of Medicine and Dentistry, Queen Mary University of London, EC1M $6 B Q$

${ }^{2}$ Barts Health NHS Trust, London, E1 $1 B B$

${ }^{3}$ NHS Blood and Transplant, Colindale, NW9 5BG

${ }^{4}$ University Hospital of Wales, Cardiff and Vale University Health Board, CF14 4XW

${ }^{5}$ Department of Haematology, Oxford University Hospitals NHS Foundation Trust, Oxford ${ }^{6}$ Radcliffe Department of Medicine, University of Oxford, and Oxford BRC Haematology Theme, Oxford, OX3 9DU

${ }^{7}$ Hampshire Hospitals NHS Foundation Trust, RG24 9NA

${ }^{8}$ Salisbury NHS Foundation Trust, SP2 8BJ

${ }^{9}$ Barking, Havering and Redbridge University Hospitals NHS Trust, RM7 0AG

${ }^{10}$ Transfusion Medicine, NHS Blood and Transplant, Oxford;

${ }^{11}$ Glasgow Royal Infirmary, NHS Greater Glasgow and Clyde, G4 OSF

\section{Address for Correspondence:}

Dr Laura Green

The Royal London Hospital

4th Floor, Pathology and Pharmacy Building

80 Newark Street

London

E1 2ES

Phone: +44 2089572756

Fax: +442089572838

Email: laura.green@bartshealth.nhs.uk

Short title: Management of DOAC-related bleeding in UK 


\section{Summary}

The lack of antidotes for the factor Xa-inhibitor direct-oral-anticoagulants (DOACs) means that management of bleeding will consist largely of existing supportive therapies. This study aimed to: a) examine the relative frequency of DOAC-related major bleeding in relation to DOACs prescriptions over study period; b) describe the presentation and haematological management of DOAC-related major bleeding; and c) evaluate the association between use of prothrombin-complex-concentrate (PCC) and in-hospital mortality. Over a three-year period, 32 UK hospitals submitted data on haematological management of DOAC-related bleeding. Results: Data consisted of 421 episodes $(67 \%, 21 \%, 11 \%$ and $1 \%$ on rivaroxaban, apixaban, dabigatran and edoxaban respectively) of major bleeding on DOACs. The proportion of major bleeds on DOACs and DOAC prescriptions increased throughout the study. Overall, $44 \%$ and $37 \%$ of patients presented with gastrointestinal bleeding and intracranial-haemorrhage (ICH) respectively. Drug concentrations were seldom measured. Compared to no PCC, there was a borderline evidence that receiving low dose PCC $(\leq 25 \mathrm{IU} / \mathrm{Kg})$ was associated with better outcomes in terms of mortality (subdistribution hazard ratio [SDHR]: 0.15; 95\%CI: 0.02-1.19; $\mathrm{p}=0.07$ ): but this was not the case for higher doses. Conclusion: DOAC concentrations are seldom measured. There was no evidence of benefit for PCC on in-hospital mortality.

Keywords: direct-oral anticoagulants, haematological management, prothrombin complex concentrate. outcome 


\section{Introduction}

It is estimated that $1.2 \%$ of the UK population are on oral anticoagulant agents (OACs)(NICE 2012). Increasing evidence on their effectiveness, coupled with an ageing population, is predicted to bring about a rise in $\mathrm{OAC}$ use. For many years warfarin (a vitamin $\mathrm{K}$ antagonist) has been the mainstay of OAC treatment; however, from 2012 onward several direct OACs (DOACs) were progressively introduced into clinical practice in the UK for stroke prevention in atrial fibrillation and the treatment and secondary prevention of venous thromboembolism (VTE). These included dabigatran etexilate (a direct thrombin inhibitor), rivaroxaban, apixaban and edoxaban (direct factor Xa inhibitors).

The development of major bleeding, particularly intracranial haemorrhage (ICH), is the most serious adverse effect of OACs, leading to significant morbidity and mortality (Chai-Adisaksopha, et al 2014, Green, et al 2018). Unlike warfarin, whose anticoagulant effect can be rapidly and completely reversed by 4-factor prothrombin complex concentrate (PCC), the lack of an antidote for the DOACs in the event of major bleeding or need for urgent surgery raised concerns for clinicians when they were introduced into clinical practice. Since there is little evidence to guide the management of major bleeding on DOACs, clinicians will use supportive measures, such as transfusion of blood components and/or administration of other haemostatic agents, as well as surgical/radiological intervention. In the absence of specific antidotes, studies in healthy volunteers have shown that PCC partially reverses the depressed endogenous thrombin potential induced by DOACs (Eerenberg, et al 2011, Marlu, et al 2012), and therefore its use is recommended by national and international guidelines (Keeling, et al 2016, Tomaselli, et al 2017).

Between 2015 and 2016 a monoclonal antibody antidote for dabigatran (idarucizumab) received regulatory approval in USA and Europe for treatment of major bleeding (2015). An antidote to the factor Xa inhibitors (andexanet alfa) has been licensed in the USA but not yet in Europe. However, the studies of idarucizumab and andexanet alfa did not include a placebo arm (Connolly, et al 2016, Pollack, et al 2017). Since the availability and/or utility of the antidotes is uncertain and they are likely to be costly it is important to establish the outcomes of DOAC-related major bleeding events in the preantidote era, so as to better evaluate their cost effectiveness for the future.

Previously we have described the bleeding characteristics and outcomes of major haemorrhage associated with all available OACs (warfarin and DOACs) in a general UK population, and identified the risk factors associated with mortality which were ICH, advanced age, spontaneous bleeding, liver failure and cancer (Green, et al 2018). In this study we further analyzed the DOAC subgroup by: a) comparing the relative share of DOAC-related major bleeding reported to this study against general OAC prescription patterns across England during the same time period; b) describing the coagulation 
abnormalities and haematological management of major bleeding on DOACs; and c) assessing the association between PCC and patients' outcomes (time to death).

\section{Methods}

Details of study approvals, case definition, identification and validation have been described previously (ORANGE study) (Green, et al 2018). In summary, over a 3-year period (October 2013 to August 2016) data on hospital admissions for major bleeding on OAC were obtained from 32 UK hospitals using patients' hospital case notes. Data comprised information on co-morbidities, bleeding sites, haematological laboratory results, management of bleeding and first outcome up to 30 days (death, discharge or continued hospitalization). Patients were not approached by the research teams and their management was not altered for the purpose of this study.

Bleeding sites were categorized into intracranial (intracerebral, subarachnoid and subdural bleeding), upper and lower gastrointestinal and "Other" (visceral, genitourinary, musculoskeletal and everywhere else); patients with multiple sites of bleeding would be assigned to a single category according to the above-stated order. Patients' laboratory results corresponding to two time-points were obtained: (T1) at clinical diagnosis of bleeding following admission and prior to treatment by hemostatic agents (such as PCC) or blood transfusion, and up to 1 day after admission; and (T2) as soon as possible after any administration of haemostatic agents or blood transfusion, and within 2 days from admission for bleeding. If no intervention was given, the earliest available result within 2 days from admission was collected. Tests included haemoglobin, platelet count, prothrombin time (PT), activated partial thromboplastin time (APTT), thrombin time (TT) and Clauss fibrinogen, as well as specific drug concentrations in plasma derived from assays such as dilute thrombin time (Hemoclot), ecarin clotting time (ECT) and anti-Xa activity.

Information on transfusion of any blood components (i.e. red blood cells [RBC], fresh frozen plasma [FFP], platelets and cryoprecipitate) for the management of bleeding was collected for up to 1 day after admission for bleeding. PCC administration was analyzed based on the patient's weight and it has been categorized into None, Low ( $\leq 25 \mathrm{IU} / \mathrm{kg})$, Medium (26- 49IU/kg) and High ( $\geq 50 \mathrm{IU} / \mathrm{kg})$. Administration of other haemostatic agents, including recombinant activated factor VII (rFVIIa), factor eight inhibitor bypassing activity (FEIBA), fibrinogen concentrate $(\mathrm{FgC})$, tranexamic acid and vitamin $\mathrm{K}$, was also recorded.

NHS England clinical commissioning groups (CCGs) prescribing data for warfarin and DOACs (rivaroxaban, dabigatran, apixaban and edoxaban) were analysed for eight quarterly periods over two years (July 2014 to June 2016). The prescribing data provided the total quantity (in $\mathrm{mg}$ ) of each OAC 
prescribed every quarter (number of items x drug content per item); the number of patients prescribed that drug during that time interval was estimated by dividing the total quantity prescribed by the Defined Daily Dose (DDD) of the OAC. The DDD is the assumed average maintenance dose per day for a drug used for its main indication in adults (available at https://www.whocc.no/atc_ddd_index/). The number of major bleeds on OAC for each period was obtained directly from this study's records. DDD for the four DOACs were combined and analyzed as a proportion of total OAC prescriptions (DOAC plus vitamin $\mathrm{K}$ antagonists).

\section{Statistical Analysis}

Variables were summarized with frequencies/proportions, means/standard deviations or median/ interquartile ranges, as appropriate. Comparisons between groups were made using Fisher's exact test, T-tests, ANOVA or non-parametric equivalents; all tests were two-sided at $5 \%$ level of significance. The effect of PCC dose on times to death was investigated using multivariable competing-risk models developed by Fine and Gray (Fine and Gray 1999) to estimate adjusted subdistribution hazard ratios (SDHR) and calculate the cumulative incidence of death. Hospital discharge was treated as a competing event since discharged patients are precluded from being able to experience a death outcome in hospital and more importantly the assumption of non-informative censoring (i.e. they have the same future risk of death as patients remaining in hospital) required by traditional survival analysis does not hold (an important premise is that patients were only discharged after they had been successfully treated for major bleeding). For comparison, standard Cox proportional hazards models were also used to estimate cause-specific hazard ratios (CSHR); these quantify the association between risk factors and death in a hypothetical world where competing events do not exist. Patients remaining hospitalized at 30 days, transferred to another hospital or lost to follow-up were censored on their last date known alive. Half a day of follow-up was added to those who died on the same day as admission for bleeding, so that all cases could be included in the analysis. Adjusted analysis using multivariable models included factors of a priori interest (type of DOAC, administration of PCC and tranexamic acid). Univariable analysis was used to select other variables judged to be predictive of the outcome, such as bleeding site (intracranial or not), red cell and plasma transfusion (as proxies for bleeding severity), co-morbidities (age, cancer, liver or renal disease, diabetes, previous stroke, congestive heart failure and ischaemic heart disease) and development of any severe complication (intensive treatment unit (ITU) admission, put on ventilator or acute respiratory distress syndrome (ARDS)). The proportional hazards assumption was tested for each covariate through fitting an interaction with time variable. All analyses were performed using STATA version 14 (StataCorp LP, USA).

\section{Results}


A total of 421 DOAC-related major bleeding episodes were reported, with $283(67 \%), 89$ (21\%), 46 $(11 \%)$ and $3(0.7 \%)$ being associated with rivaroxaban, apixaban, dabigatran and edoxaban, respectively. Aggregating across all NHS CCGs in England, whose catchment makes up 84\% of the adult population ( $\geq 20$ years) in the UK (Office for National Statistics licensed under the Open Government Licence - Population Estimates for UK, England and Wales, Scotland and Northern Ireland: Mid-2016), the proportion of orally anticoagulated patients on a DOAC each quarter increased almost linearly between July 2014 and June 2016 (Figure 1). The proportion of DOAC major bleeds reported to ORANGE study showed a similar, albeit non-linear, increase within the same period but remained for the most part under the level of DOAC prescriptions.

Figure 1. Percentage of DOACs (NHS CCGs) and DOAC major bleeding events (ORANGE) against all OAC prescriptions/bleeds, by quarter

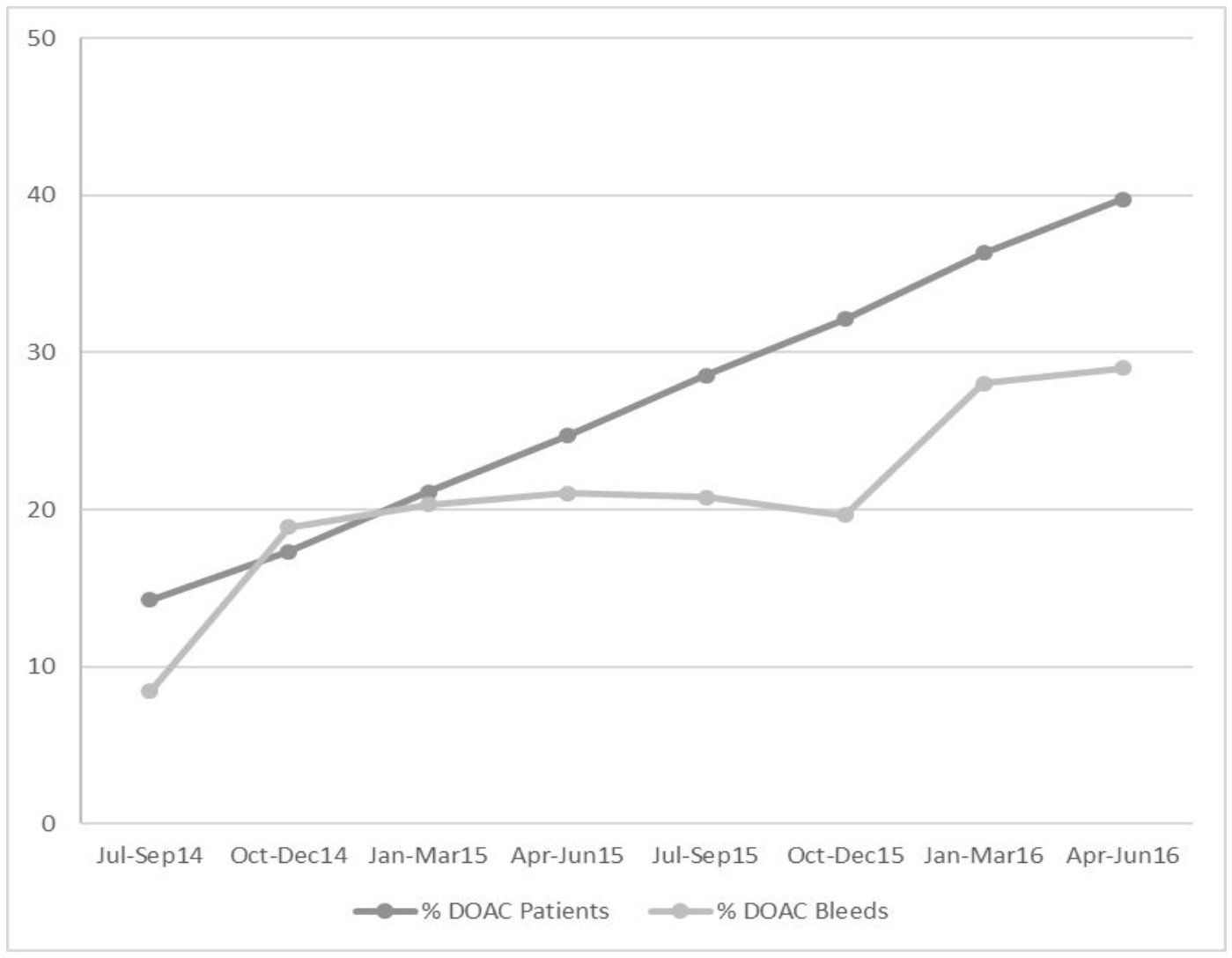

DOACs: direct oral anticoagulant agents; NHS CCG: National Health Service clinical commissioning group; ORANGE: ORal ANticoagulant aGEent-associated bleeding events reporting system 
The characteristics of patients on DOACs at admission for major bleeding have been described previously (Green, et al 2018). In summary, the median (IQR) age was 83 (75-88) years, with no significant differences between DOACs. There were proportionally more atrial fibrillation patients on dabigatran and apixaban compared with rivaroxaban (partially reflecting the earlier licensing of rivaroxaban compared to the other DOACs for VTE treatment in the UK), and there was a greater prevalence of chronic kidney disease and a history of stroke amongst rivaroxaban and dabigatran patients respectively, compared to other DOACs. Overall 155 (37\%), 186 (44\%) and 80 (19\%) patients presented with intracranial haemorrhage (ICH), gastro-intestinal bleeding (GI) and Other (non-ICH or GI bleed) respectively (Table 1). GI bleeding made up the majority of bleeds on both dabigatran and rivaroxaban, whilst almost half of apixaban bleeds were $\mathrm{ICH}$.

Table 1. Description of Bleed Sites by direct oral anticoagulants ${ }^{\#}$

\begin{tabular}{|c|c|c|c|c|c|c|c|c|c|c|}
\hline \multirow[b]{2}{*}{ Intracranial } & \multicolumn{2}{|c|}{$\begin{array}{c}\text { Dabigatran } \\
\quad N=46\end{array}$} & \multicolumn{2}{|c|}{$\begin{array}{c}\text { Rivaroxaban } \\
\mathbf{N}=\mathbf{2 8 3}\end{array}$} & \multicolumn{2}{|c|}{$\begin{array}{c}\text { Apixaban } \\
\mathbf{N}=\mathbf{8 9} \\
\text { n }(\%)\end{array}$} & \multicolumn{2}{|c|}{$\begin{array}{c}\text { Edoxaban } \\
\mathbf{N}=\mathbf{3}\end{array}$} & \multicolumn{2}{|c|}{$\begin{array}{l}\text { Overall } \\
N=421\end{array}$} \\
\hline & 14 & $(30.4)$ & 97 & $(34.3)$ & 43 & $(48.3)$ & 1 & $(33.3)$ & 155 & $(36.8)$ \\
\hline Intracerebral & 10 & $(21.7)$ & 59 & $(20.8)$ & 24 & $(27.0)$ & 1 & $(33.3)$ & 94 & $(22.3)$ \\
\hline Subarachnoid & 1 & $(2.2)$ & 10 & (3.5) & 8 & $(9.0)$ & 0 & $(0.0)$ & 19 & $(4.5)$ \\
\hline Subdural & 3 & $(6.5)$ & 28 & $(9.9)$ & 11 & $(12.4)$ & 0 & $(0.0)$ & 42 & $(10.0)$ \\
\hline Gastrointestinal (GI) & 27 & $(58.7)$ & 127 & $(44.9)$ & 31 & $(34.8)$ & 1 & $(33.3)$ & 186 & $(44.2)$ \\
\hline Upper GI & 12 & $(26.1)$ & 75 & $(26.5)$ & 19 & $(21.3)$ & 1 & $(33.3)$ & 107 & $(25.4)$ \\
\hline Lower GI & 15 & $(32.6)$ & 52 & $(18.4)$ & 12 & $(13.5)$ & 0 & $(0.0)$ & 79 & $(18.8)$ \\
\hline Other* & 5 & $(10.9)$ & 59 & $(20.8)$ & 15 & (16.9) & 1 & $(33.3)$ & 80 & $(19.0)$ \\
\hline Visceral & 1 & $(2.2)$ & 14 & (4.9) & 4 & $(4.5)$ & 0 & $(0.0)$ & 19 & $(4.5)$ \\
\hline Genitourinary & 1 & $(2.2)$ & 13 & $(4.6)$ & 0 & $(0.0)$ & 1 & (33.3) & 15 & $(3.6)$ \\
\hline Musculoskeletal & 1 & $(2.2)$ & 26 & $(9.2)$ & 6 & (6.7) & 0 & $(0.0)$ & 33 & $(7.8)$ \\
\hline Miscellaneous & 2 & $(4.3)$ & 6 & $(2.1)$ & 5 & (5.6) & 0 & $(0.0)$ & 13 & $(3.1)$ \\
\hline
\end{tabular}

\# Patients reported to have more than one bleeding site were assigned to the clinically most severe, in descending order of priority as above.

* Explanation of Other

Visceral Haemoptysis; Pericardium; Retroperitoneal; Abdomen; Chest/Thoracic

Genitourinary Haematuria/Urethral; Vaginal

Musculoskeletal Epistaxis or mucosal; Cutaneous or soft tissue; Intra-articular; Oral/Pharyngeal

Miscellaneous Surgical site; Intraocular; Puncture site; Unknown; Other not covered above

(cases with more than 1 of the above classified in descending order of priority) 


\section{Laboratory results}

There was considerable variation in the completeness of test results available at $\mathrm{T} 1$ and $\mathrm{T} 2$. Overall, $96 \%$ of patients had haemoglobin and platelet count measured at T1, and just over $65 \%$ had these tests at T2. The coagulation assays on the other hand were done on fewer patients: at $\mathrm{T} 165 \%, 82 \%$ and $50 \%$ had PT, APTT and fibrinogen measured respectively, and at T2 the test rates decreased to $37 \%, 46 \%$ and $26 \%$ respectively. The greater percentage of missing results at $\mathrm{T} 2$ can be partly explained by the fact that about $10 \%$ of patients died within two days. Drug concentrations were seldom measured; this was particularly lacking for post-intervention monitoring. The hemoclot test was performed in 1 out of 46 dabigatran patients at $\mathrm{T} 1$ but not at $\mathrm{T} 2$; ecarin clotting time was never done. Rivaroxaban anti-Xa assay was performed in $23(8 \%)$ patients at $\mathrm{T} 1$ (median [IQR]: 111 [41-360] ng/mL) and $11(4 \%)$ at T2 $(65$ [34-163] ng/mL). Apixaban anti-Xa assay was performed in 10 (11\%) patients at T1 (median [IQR]: $231[133-262] \mathrm{ng} / \mathrm{mL})$ and only $2(1 \%)$ at $\mathrm{T} 2(103[102-104] \mathrm{ng} / \mathrm{mL})$.

There was no difference in haemoglobin, platelet count and fibrinogen levels at both $\mathrm{T} 1$ and $\mathrm{T} 2$ between DOACs. The median PT was more prolonged for dabigatran and rivaroxaban (at both time points) than apixaban, while APTT (at both time points) was more prolonged with dabigatran than other DOACs (Figure 2). Analysis of a subgroup with results at both T1 and T2 showed the median PT $(n=146)$ and APTT $(\mathrm{n}=180)$ were $\sim 4$ seconds higher at T1 than T2 across all DOACs; the differences were statistically significant $(\mathrm{p}<0.001$ for both). 
Figure 2. Laboratory results at admission (T1) and post-intervention (T2), by direct oral anticoagulant

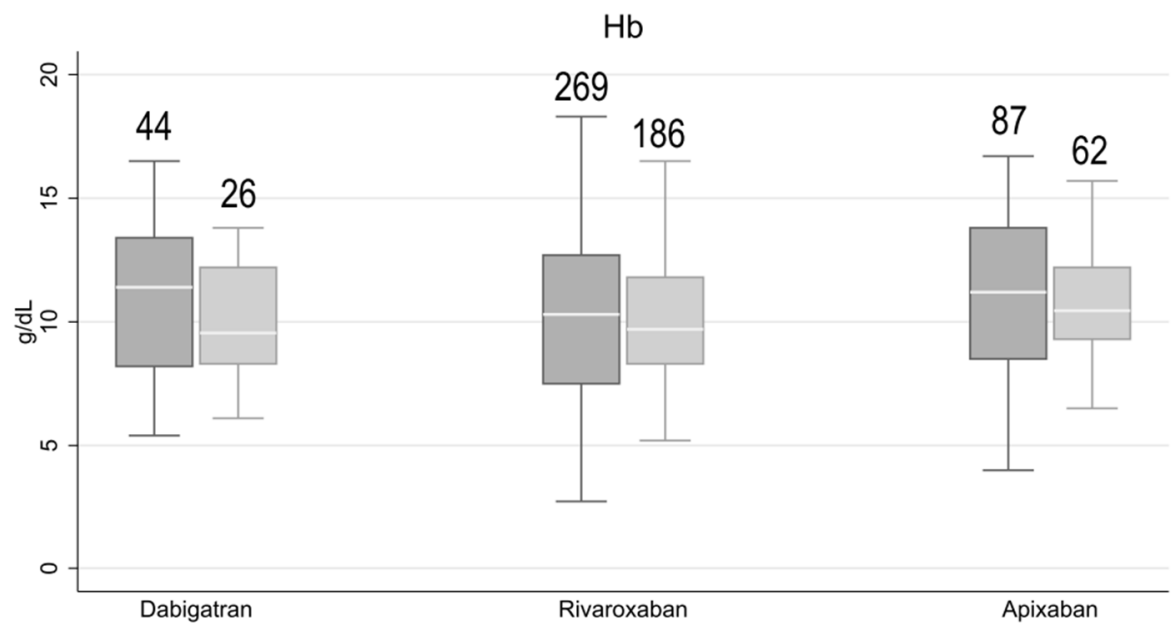

PT
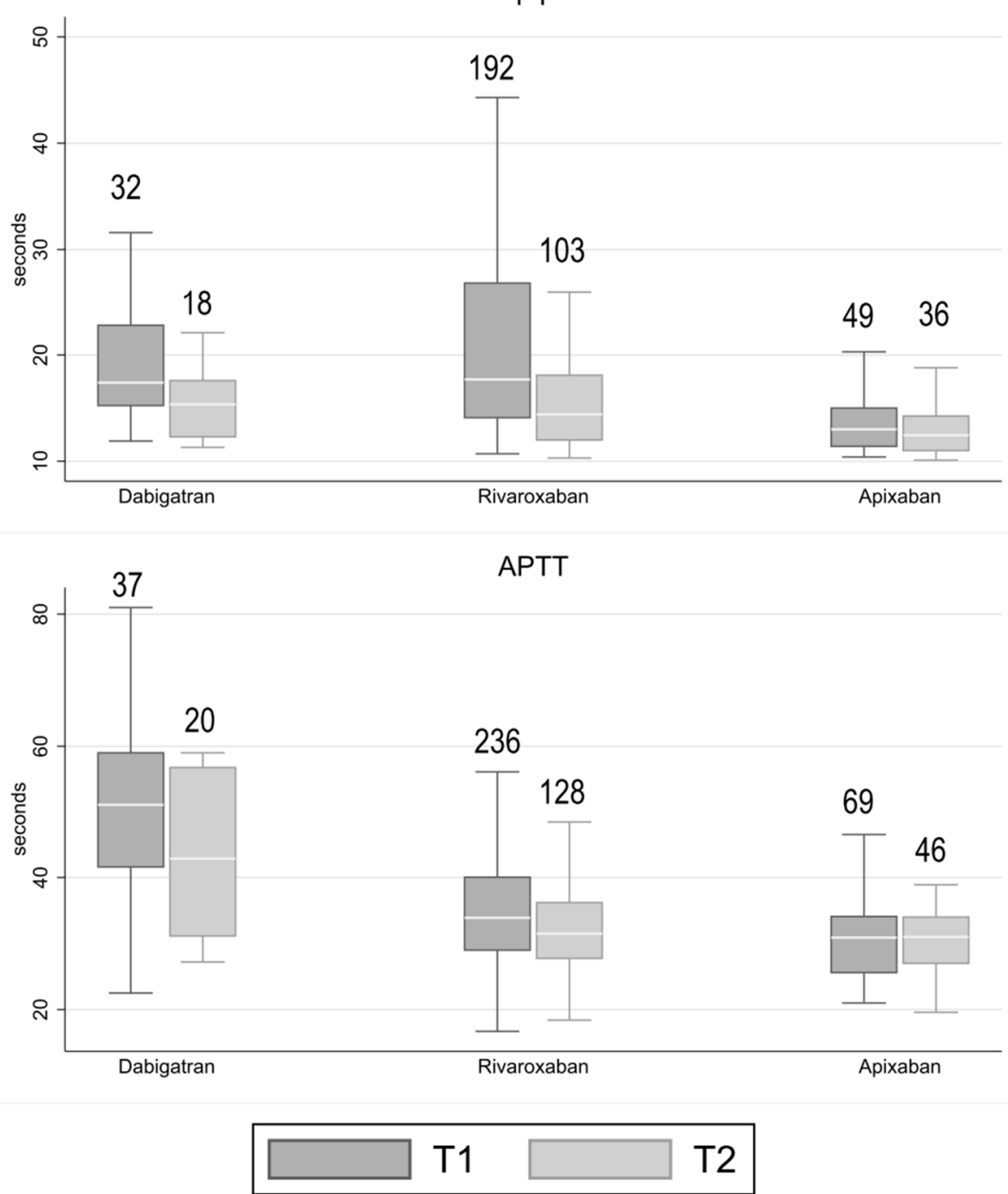

Hb: haemoglobin; PT: prothrombin time; APTT: activated partial thromboplastin time.

Numbers above boxes denote number of observations. Values beyond ( $1.5 \mathrm{x}$ interquartile range) from the lower and upper quartiles not shown. Edoxaban not plotted. 


\section{Haematological management of bleeding and outcome}

Transfusion of blood components and/or haemostatic agents was given to 301 (72\%) patients within 24 hours of bleeding. Overall $41 \%, 38 \%$ and $28 \%$ of patients received any blood component, PCC and tranexamic acid, respectively (Table 3 ).

Table 3: $\quad$ Haematological management of bleeding by DOACs, number (\%)

\begin{tabular}{lccccc} 
& $\begin{array}{c}\text { Dabigatran } \\
\mathrm{N}=46\end{array}$ & $\begin{array}{c}\text { Rivaroxaban } \\
\mathrm{N}=283\end{array}$ & $\begin{array}{c}\text { Apixaban } \\
\mathrm{N}=89\end{array}$ & $\begin{array}{c}\text { Edoxaban } \\
\mathrm{N}=3\end{array}$ & $\begin{array}{c}\text { Overall } \\
\mathrm{N}=421\end{array}$ \\
Received any intervention & $31(67)$ & $193(68)$ & $75(84)$ & $2(67)$ & $301(72)$ \\
& & & & & \\
PCC & $11(24)$ & $104(37)$ & $45(51)$ & $2(67)$ & $162(38)$ \\
Tranexamic Acid & $12(26)$ & $73(26)$ & $29(33)$ & $2(67)$ & $116(28)$ \\
Vitamin K & $9(20)$ & $41(14)$ & $14(16)$ & 0 & $64(15)$ \\
FEIBA & $3(6)$ & $1(<1)$ & $1(1)$ & 0 & $5(1)$ \\
& $22(48)$ & $117(41)$ & $32(36)$ & $1(33)$ & $172(41)$ \\
Any blood transfusion & $16(35)$ & $112(40)$ & $29(33)$ & $1(33)$ & $158(38)$ \\
Red Blood Cells & $5(11)$ & $18(6)$ & $8(9)$ & 0 & $31(7)$ \\
Fresh Frozen Plasma & $5(11)$ & $8(3)$ & $5(6)$ & 0 & $18(4)$ \\
Platelets & $2(4)$ & $3(1)$ & $2(2)$ & 0 & $7(2)$ \\
Cryoprecipitate & & & & & \\
\hline
\end{tabular}

DOAC: direct oral anticoagulant agents; PCC: prothrombin complex concentrate; FEIBA: factor eight inhibitor bypassing activity.

In the final 8 months of the study, 7 patients on dabigatran received idarucizumab for dabigatran reversal and 5 apixaban patients were given andexanet alfa as part of a clinical trial. PCC was more commonly administered to patients with ICH (50\%) than those with non-ICH bleeds (32\%). Overall, rivaroxaban and apixaban patients were more likely to be given PCC than dabigatran patients. Analysis of 108 (67\%) patients who received PCC with weight recorded showed considerable variation in weight for selected fixed PCC doses (Figure 3); the median (IQR) dosing regimen was 35 (25-49) IU/kg with 25 and 50 $\mathrm{IU} / \mathrm{kg}$ being the most common.

In total five $(1.2 \%)$ and three $(0.7 \%)$ patients developed a venous and arterial thromboembolic event respectively during their hospital stay but there was no evidence that thromboembolic events were associated with PCC administration ( $\mathrm{p}=0.49)$. Of 413 (98\%) patients who were followed-up until discharge, death or 30 days (whichever occurred first), 88 (21.3\%; 95\% CI: $17.5-25.6 \%)$ had died in hospital within 30 days, with no significant differences between the DOACs $(p=0.66)$. Amongst discharged patients, the median (IQR) stay in hospital was 6 (3-11) days (data not shown); this was not different between DOACs $(\mathrm{p}=0.24)$. 
Figure 3: Distribution of total Prothrombin Complex Concentrate (PCC) dose for patients who received PCC within one day of admission for major bleeding and whose weight was known ( $\mathrm{n}=108)$.

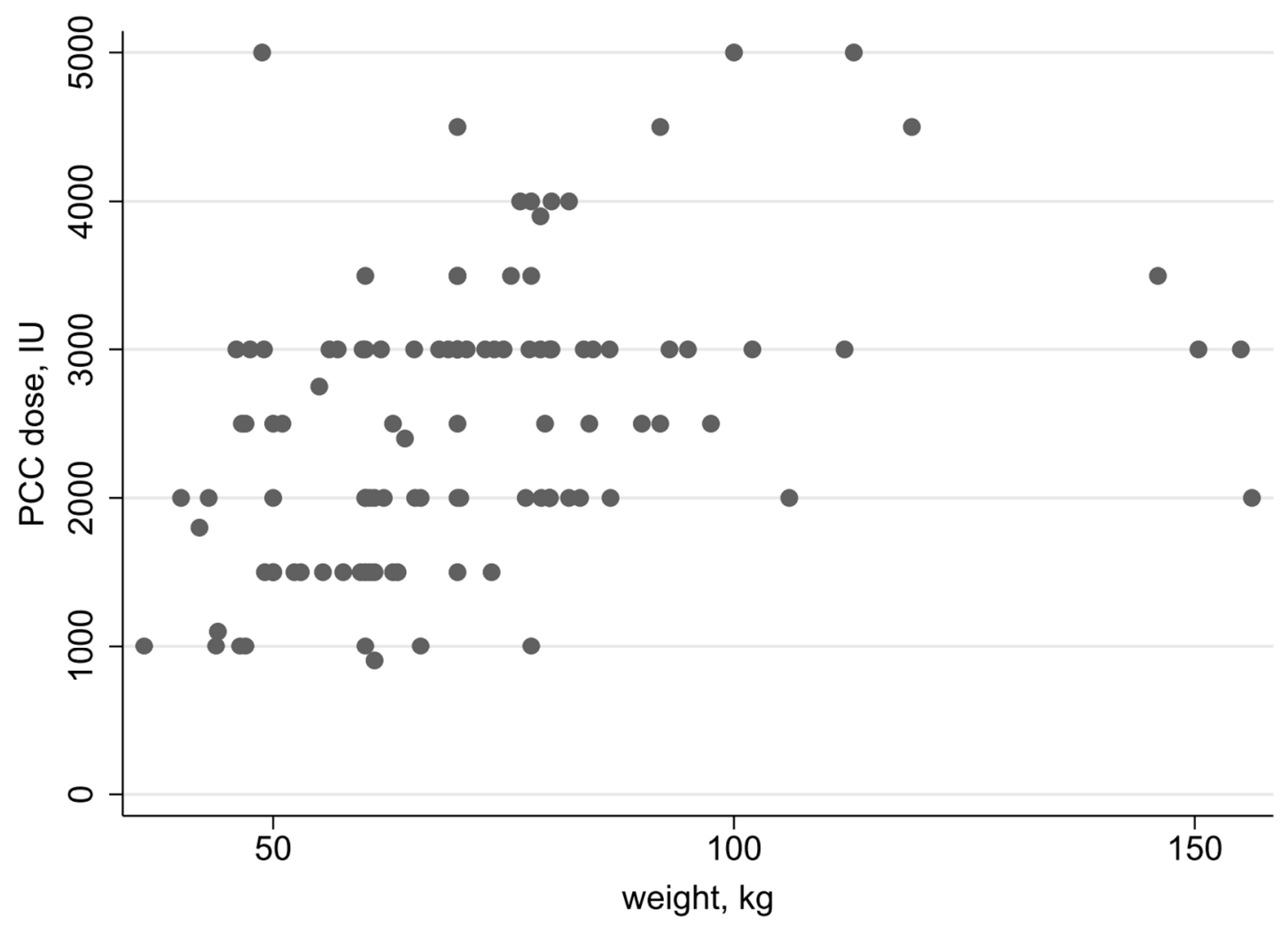

One point with PCC dose of 10,000 IU not plotted.

\section{Analysis of reversal strategies and time to death}

Overall $20 \%$ and $4.5 \%$ of ICH and non-ICH patients died within 2 days of admission respectively. Time to death was right-skewed with a median (IQR) of 3 (1-9) days; $54 \%$ and $40 \%$ of deaths in the ICH and non-ICH groups respectively occurred within two days after admission. Analysis of 367 patients with known PCC dose regimens showed that compared with not receiving PCC, getting $\leq 25 \mathrm{IU} / \mathrm{kg}, 26-$ $49 \mathrm{IU} / \mathrm{kg}$ and $\geq 50 \mathrm{IU} / \mathrm{kg}$ of PCC were not significantly predictive of the cumulative risk of death; however there is arguably borderline evidence of the low dose regimen being associated with better outcomes. The presence of ICH and history of previous stroke increased the cumulative incidence of death by 3.35 times $(95 \% \mathrm{CI}: 1.72-6.52 ; \mathrm{p}<0.001)$ and 2.13 times $(95 \% \mathrm{CI}: 1.24-3.65 ; \mathrm{p}=0.006)$ respectively (Table 4), whilst the type of DOAC, administration of tranexamic acid and total red cell+plasma transfusion (as proxy for blood loss) were not found to be associated with risk of death. There was no evidence that the proportional hazards assumption was violated for any of the risk factors in the model. Lastly, the SDHRs were in general slightly greater than the corresponding cause-specific hazard ratios (CSHRs) for all risk factors, but did not differ materially in terms of significance. 
Table 4: Multivariable Analysis for Time to Death: Subdistribution-Hazard Ratio (SDHR) estimated using Competing-risks methods (Fine \& Gray)

\begin{tabular}{lllll}
\hline Covariate & SDHR & \multicolumn{2}{c}{$\mathbf{9 5 \% C I}$} & p-value \\
\hline No PCC & 1 & & & \\
$\quad$ Low $(\leq 25 \mathrm{IU} / \mathrm{kg})$ & 0.15 & 0.02 & 1.19 & 0.073 \\
Medium $(26-49 \mathrm{IU} / \mathrm{kg})$ & 1.21 & 0.63 & 2.34 & 0.571 \\
High ( $\geq 50 \mathrm{IU} / \mathrm{kg})$ & 2.02 & 0.89 & 4.56 & 0.092 \\
Tranexamic Acid & 0.90 & 0.49 & 1.68 & 0.746 \\
ICH & 3.35 & 1.72 & 6.52 & $<0.001$ \\
Red Cell/Plasma received (unit) & 0.97 & 0.81 & 1.17 & 0.755 \\
Previous stroke & 2.13 & 1.24 & 3.65 & 0.006 \\
ITU/ARDS/Ventilation & 3.16 & 1.74 & 5.72 & $<0.001$ \\
& & & &
\end{tabular}

Estimates adjusted for all other variables in the model

PCC: prothrombin complex concentrate; ICH: intracranial haemorrhage; ITU: intensive care unit; ARDS: acute respiratory distress syndrome

\section{Discussion}

This UK multicentre, observational study of practice has reported on the haematological management and outcome of patients who developed major bleeding associated with DOACs. Key findings are: there were no significant differences between DOACs in terms of mortality rates, or the duration of hospitalization for surviving patients; there is a general lack of post-intervention coagulation monitoring, and drug concentrations were rarely measured; PCC was the most frequently prescribed treatment, although evidence of effectiveness for this indication remains uncertain.

The prescription data showed that the use of DOACs has risen steadily over time relative to warfarin, and the corresponding increase in the proportion of DOAC-related major bleeding is an indication that our study sample is representative of the wider population. Previous studies that have reported on the real-world outcomes of major bleeding associated with DOACs have grouped these agents into a single entity (Becattini, et al 2017, Lamberts, et al 2017, Xu, et al 2017). In this study we saw variations in the distribution of bleeding sites across DOACs, with GI bleeding constituting a greater percentage of dabigatran major bleeds, whilst proportionally more apixaban bleeds were ICH. It is important to emphasize that a greater proportion of GI bleeding in dabigatran patients compared with apixaban patients does not imply that the risk of GI bleeding is higher with dabigatran than apixaban; i.e. the observed differences in bleeding sites between the different DOACs are relative rather than absolute. Nonetheless, the question of why the odds of a GI bleed would be higher on dabigatran compared to apixaban - if one were to develop bleeding - is an important one which warrants further research. 
Haematological management of patients who develop major bleeding (with or without OAC) requires that coagulation assays be performed for guiding treatment (Hunt, et al 2015, Keeling, et al 2011). This study found that specific laboratory assays for measuring drug concentrations were seldom performed. Our findings are similar to the study by Schulman and colleagues that reported a lower rate of drugspecific tests (being performed in these patients (Schulman, et al 2018). Considering that administration of blood components and other haemostatic agents is not wholly without risks, making these assays available in all laboratories is important not least to assess the need for and monitor the effect of the new targeted reversal agents which are likely to be partly guided by drug-specific coagulation assays (Weitz and Eikelboom 2016).

PCC was the most frequently prescribed treatment ( 40\%), and patients who had ICH were more likely to receive it. Compared with other studies (Becattini, et al 2017, Xu, et al 2017), the rate of PCC use in our study was higher, and this is likely due to its availability in most UK hospitals for the treatment of warfarin-related major bleeding, as well as the higher ICH rates in our study; the latter also accounts for the difference in mortality between our studies and others (Becattini, et al 2017, Lamberts, et al 2017, Xu, et al 2017). One uncertainty centres on how PCC should be dosed for the management of DOAC-related bleeding. Previous studies into PCC dosing for DOAC reversal have either involved randomized controlled trials in healthy (non-bleeding) volunteers (Barco, et al 2016, Eerenberg, et al 2011) or observational studies without controls (Majeed, et al 2017, Schulman, et al 2018). Our study found that both $25 \mathrm{IU} / \mathrm{kg}$ and $50 \mathrm{IU} / \mathrm{kg}$ were the most common regimens in our study, which suggests that some clinicians may have relied on approximate adjustments for weight. However, weight-adjusted dosing for PCC in DOAC-related bleeding is likely to have been borrowed from experience with warfarin reversal, rather than direct evidence.

Considering that DOACs are given at a fixed dose, one might justify the appropriateness of giving a fixed rather than weight-adjusted PCC dose for DOAC reversal; pharmacokinetic studies of DOAC prescriptions in healthy volunteers showed the area under the curve is unaffected by body weight for rivaroxaban (Kubitza, et al 2007) and apixaban (Upreti, et al 2013). Cohort studies of fixed-dose PCC (2,000 IU, equivalent to $25 \mathrm{IU} / \mathrm{kg}$ in a $80 \mathrm{~kg}$ patient) have found that it was "effective" in $\sim 70 \%$ of patients with ICH on rivaroxaban and apixaban; however, these studies lacked a control group needed to evaluate the effect attributable to PCC (Majeed, et al 2017, Schulman, et al 2018). Further, in these studies the measure of effectiveness was in part subjective as it relied on the treating local physician applying the ISTH (International Society of Thrombosis and Haemostasis Scientific) assessment criteria. Of note the median time from the last dose of rivaroxaban or apixaban to treatment with PCC was $>12$ hours in both studies and was therefore different from the 3 hours after rivaroxaban ingestion used in the "dose-finding" study of PCC in healthy volunteers (Barco, et al 2016). 
Our analysis showed that after adjusting for bleeding site, bleeding severity and other potential confounders, there was no statistically significant association between PCC and risk of death. One possible explanation is that PCC was usually administered as a last resort for the management of bleeding in rapidly deteriorating patients, and therefore often too late to have any effect. We did not collect the timing from the onset of bleeding to administration of PCC, which is a limitation of the study. It is also interesting to find arguably borderline evidence of a dose-response relationship, and particularly that PCC in low doses seem to be associated with better outcomes, but this was not the case with higher dose; whether this is a true effect or reverse causality can only be determined by further research. Due to drug-specific assays not being performed to estimate the amount of DOAC onboard at the time of PCC administration and weight not necessarily being up to date, there remains scope for residual confounding. We should also state the caveat that cases who received PCC but did not have weight recorded were excluded from the analysis, but the findings were nonetheless based on $87 \%$ of the sample and therefore likely to be relatively robust.

Notwithstanding the advent and licensing of new specific antidotes for reversal of DOAC activity, we believe that their relative high costs and limited availability, particularly for FXa inhibitor reversal agents, is likely to result in the continued prescription of PCC for the management of DOAC-related bleeding for some time to come. Our findings have provided the impetus and underscore the need for interventional studies to evaluate the clinical efficacy of PCC in the treatment of DOAC-related bleeding through investigating: first, if PCC should be given for management of DOAC-related bleeding; and second, if PCC should be given as a fixed dose or weight-adjusted regimen, and the appropriate dose required to reverse anticoagulation activity.

A key strength of our study is that cases have been prospectively identified in parallel with hospital admissions for major bleeding on DOACs and data have been collected directly from clinical notes, thus ensuring a high degree of fidelity with events and minimizing loss of detail associated with coding in administrative databases. Second, by circumventing patient consent the study avoided introducing selection bias and all eligible cases were included. Third, with multiple hospitals across the UK reporting to the study, our results are generalizable and allow for a deeper insight into current practices across a wide geographical area. In addition to limitations previously mentioned above, we did not establish clinicians' reasons for using PCC or if they were guided by weight in dosing. Further, we do not know why drug concentrations were not being measured - e.g. was it due to the assays not being available, or they were not requested due to lack of knowledge, or because clinicians did not think the results will help with management of bleeding? Nonetheless, our study remains the first and largest to report on the association between PCC use and mortality in patients who develop major bleeding on DOACs, and it has highlighted that the use of PCC in DOAC-related major bleeding should be guided 
by a richer knowledge base, particularly since it will likely remain part of the management strategy for the future.

\section{Funding}

The study was funded by the British Society for Haematology Early Stage Researcher Fellowship awarded to Dr Laura Green. The views expressed in this publication are those of the authors and not those of the funder. The funder had no role in the study design, data collection/analysis or preparation of this article.

\section{Acknowledgments}

We would like to acknowledge all hospitals, NHS trusts and principal investigators who reported to the study: Newham University Hospital (Dr Olivia Kreze), Whipps Cross University Hospital (Dr Peter MacCallum); The Royal London Hospital (Dr Laura Green); Barking Havering and Redbridge University Hospitals (Dr Khalid Saja); John Radcliffe Hospital (Dr Nicola Curry); Glasgow Royal Infirmary (Dr Campbell Tait); University Hospital of Wales (Dr Raza Alikhan); Sheffield Teaching Hospitals (Dr Rhona Maclean); Basingstoke \& North Hampshire Hospital and Salisbury District Hospital (Dr Tamara Everington); Ulster Hospital (Dr Margaret Bowers); Wexham Park Hospital (Dr Sarah Wilson); Withybush General Hospital (Dr Sumant Kundu); Newcastle upon Tyne Hospitals (Dr John Hanley); North Middlesex University Hospital (Dr John Luckit); Basildon and Thurrock Hospitals (Dr Godwin Simon); Torbay Hospital (Dr Nichola Rymes); University College Hospital (Dr Hannah Cohen); St George's Healthcare NHS Trust (Dr James Uprichard); Birmingham Heartlands Hospital (Dr Charalampos Kartsios); Royal Bournemouth Hospital (Dr Jason Mainwaring); Hull and East Yorkshire Hospitals NHS Trust (Dr Salama Abosaad); Northern Lincolnshire and Goole NHS Foundation Trust (Dr Sanjeev Jalihal); Dumfries and Galloway Royal Infirmary (Dr Mohamed Khan); University Hospitals of North Midlands (Dr Deepak Chandra); Gloucestershire Hospitals (Dr Oliver Miles); South Tees Hospitals (Dr Jamie Maddox); Glangwili General Hospital (Dr Saran Nicholas); Royal Berkshire NHS Foundation Trust (Dr Liza Keating); University Hospital Southampton (Dr Sara Boyce); Northumbria Healthcare NHS Foundation Trust (Dr Charlotte Bomken); and North Cumbria University Hospitals (Dr Roderick Oakes).

\section{Authorship contribution}

LG, JT, PM, and JKM designed the study, wrote the manuscript and made all subsequent revisions. JT and JM performed the statistical analysis. CT, SA, SS, KS, TE, RA, and NC contributed to the design of the study and writing of the manuscript. 


\section{Disclosure of conflict of interest}

All authors declare the following: LG, JT, JM, NC, TE and SS have no conflicts of interest to disclose. SA has received honorarium from Bayer Healthcare, Daiichi Sankyo, and Portola, outside the submitted work. RA has received honorarium from Bayer, Daiichi-Sankyo, Boehringer Ingelheim and BMS/Pfizer. KS has received honorarium from Bayer, BMS/Pfizer and Boehringer Ingelheim. CT has received honoraria and/or support to attend scientific meetings from Bayer, Pfizer, NovoNordisk, Shire, Sobi, CSL Behring and Daiichi-Sankyo. PM has served as a speaker for Bayer and Daiichi-Sankyo, as a consultant for Daiichi-Sankyo, and has received travel support from Boehringer Ingelheim. 


\section{References}

(2015) Food and Drug Administration. FDA approves Praxbind, the first reversal agent for the anticoagulant Pradaxa. Oct 16, 2015. Available at: www.fda.gov/newsevents/newsroom/pressannouncements/ucm467300.htm,

Accessed 12 January 2018.

Barco, S., Whitney Cheung, Y., Coppens, M., Hutten, B.A., Meijers, J.C. \& Middeldorp, S. (2016) In vivo reversal of the anticoagulant effect of rivaroxaban with four-factor prothrombin complex concentrate. Br J Haematol, 172, 255-261.

Becattini, C., Franco, L., Beyer-Westendorf, J., Masotti, L., Nitti, C., Vanni, S., Manina, G., Cattinelli, S., Cappelli, R., Sbrojavacca, R., Pomero, F., Marten, S. \& Agnelli, G. (2017) Major bleeding with vitamin $\mathrm{K}$ antagonists or direct oral anticoagulants in real-life. Int $\mathrm{J}$ Cardiol, 227, 261-266.

Chai-Adisaksopha, C., Crowther, M., Isayama, T. \& Lim, W. (2014) The impact of bleeding complications in patients receiving target-specific oral anticoagulants: a systematic review and meta-analysis. Blood, 124, 2450-2458.

Connolly, S.J., Milling, T.J., Jr., Eikelboom, J.W., Gibson, C.M., Curnutte, J.T., Gold, A., Bronson, M.D., Lu, G., Conley, P.B., Verhamme, P., Schmidt, J., Middeldorp, S., Cohen, A.T., Beyer-Westendorf, J., Albaladejo, P., Lopez-Sendon, J., Goodman, S., Leeds, J., Wiens, B.L., Siegal, D.M., Zotova, E., Meeks, B., Nakamya, J., Lim, W.T., Crowther, M. \& Investigators, A.-. (2016) Andexanet Alfa for Acute Major Bleeding Associated with Factor Xa Inhibitors. N Engl J Med, 375, 1131-1141.

Eerenberg, E.S., Kamphuisen, P.W., Sijpkens, M.K., Meijers, J.C., Buller, H.R. \& Levi, M. (2011) Reversal of rivaroxaban and dabigatran by prothrombin complex concentrate: a randomized, placebo-controlled, crossover study in healthy subjects. Circulation, 124, 1573-1579.

Fine, J.P. \& Gray, R.J. (1999) A Proportional Hazards Model for the Subdistribution of a Competing Risk. Journal of the American Statistical Association, 94, 496-509.

Green, L., Tan, J., Morris, J.K., Alikhan, R., Curry, N., Everington, T., McLean, R., Saja, K., Stanworth, S., Tait, C. \& MacCallum, P. (2018) A three-year prospective study of the presentation and clinical outcomes of major bleeding episodes associated with oral anticoagulant use in the UK (ORANGE study). Haematologica.

Hunt, B.J., Allard, S., Keeling, D., Norfolk, D., Stanworth, S.J. \& Pendry, K. (2015) A practical guideline for the haematological management of major haemorrhage. Br. J. Haematol.

Keeling, D., Baglin, T., Tait, C., Watson, H., Perry, D., Baglin, C., Kitchen, S. \& Makris, M. (2011) Guidelines on oral anticoagulation with warfarin - fourth edition. Br. J. Haematol, 154, 311-324.

Keeling, D., Tait, R.C., Watson, H. \& British Committee of Standards for, H. (2016) Perioperative management of anticoagulation and antiplatelet therapy. Br J Haematol, 175, 602-613.

Kubitza, D., Becka, M., Zuehlsdorf, M. \& Mueck, W. (2007) Body weight has limited influence on the safety, tolerability, pharmacokinetics, or pharmacodynamics of rivaroxaban (BAY 59-7939) in healthy subjects. J Clin Pharmacol, 47, 218-226.

Lamberts, M., Staerk, L., Olesen, J.B., Fosbol, E.L., Hansen, M.L., Harboe, L., Lefevre, C., Evans, D. \& Gislason, G.H. (2017) Major Bleeding Complications and Persistence With Oral Anticoagulation in Non-Valvular Atrial Fibrillation: Contemporary Findings in RealLife Danish Patients. J Am Heart Assoc, 6. 
Majeed, A., Agren, A., Holmstrom, M., Bruzelius, M., Chaireti, R., Odeberg, J., Hempel, E.L., Magnusson, M., Frisk, T. \& Schulman, S. (2017) Management of rivaroxaban- or apixaban-associated major bleeding with prothrombin complex concentrates: a cohort study. Blood, 130, 1706-1712.

Marlu, R., Hodaj, E., Paris, A., Albaladejo, P., Cracowski, J.L. \& Pernod, G. (2012) Effect of non-specific reversal agents on anticoagulant activity of dabigatran and rivaroxaban: a randomised crossover ex vivo study in healthy volunteers. Thromb Haemost, 108, 217-224.

NICE (2012) Assumptions used in estimating a population benchmark. http://www. nice. org. uk/usingguidance/commissioningguides/anticoagulationtherapyservice/popbench. jsp.

Pollack, C.V., Jr., Reilly, P.A., van Ryn, J., Eikelboom, J.W., Glund, S., Bernstein, R.A., Dubiel, R., Huisman, M.V., Hylek, E.M., Kam, C.W., Kamphuisen, P.W., Kreuzer, J., Levy, J.H., Royle, G., Sellke, F.W., Stangier, J., Steiner, T., Verhamme, P., Wang, B., Young, L. \& Weitz, J.I. (2017) Idarucizumab for Dabigatran Reversal - Full Cohort Analysis. N Engl J Med, 377, 431-441.

Schulman, S., Gross, P.L., Ritchie, B., Nahirniak, S., Lin, Y., Lieberman, L., Carrier, M., Crowther, M.A., Ghosh, I., Lazo-Langner, A., Zondag, M. \& Study, I. (2018) Prothrombin Complex Concentrate for Major Bleeding on Factor Xa Inhibitors: A Prospective Cohort Study. Thromb Haemost, 118, 842-851.

Tomaselli, G.F., Mahaffey, K.W., Cuker, A., Dobesh, P.P., Doherty, J.U., Eikelboom, J.W., Florido, R., Hucker, W., Mehran, R., Messe, S.R., Pollack, C.V., Jr., Rodriguez, F., Sarode, R., Siegal, D. \& Wiggins, B.S. (2017) 2017 ACC Expert Consensus Decision Pathway on Management of Bleeding in Patients on Oral Anticoagulants: A Report of the American College of Cardiology Task Force on Expert Consensus Decision Pathways. J Am Coll Cardiol, 70, 3042-3067.

Upreti, V.V., Wang, J., Barrett, Y.C., Byon, W., Boyd, R.A., Pursley, J., LaCreta, F.P. \& Frost, C.E. (2013) Effect of extremes of body weight on the pharmacokinetics, pharmacodynamics, safety and tolerability of apixaban in healthy subjects. $\mathrm{Br} J \mathrm{Clin}$ Pharmacol, 76, 908-916.

Weitz, J.I. \& Eikelboom, J.W. (2016) Urgent Need to Measure Effects of Direct Oral Anticoagulants. Circulation, 134, 186-188.

Xu, Y., Schulman, S., Dowlatshahi, D., Holbrook, A.M., Simpson, C.S., Shepherd, L.E., Wells, P.S., Giulivi, A., Gomes, T., Mamdani, M., Khuu, W., Frymire, E., Johnson, A.P. \& Bleeding Effected by Direct Oral Anticoagulants Study, G. (2017) Direct Oral Anticoagulant- or Warfarin-Related Major Bleeding: Characteristics, Reversal Strategies, and Outcomes From a Multicenter Observational Study. Chest, 152, 8191. 